\title{
Developing of Fuzzy Logic Decision Support for Management of Breast Cancer
}

\author{
Sameh Mohamed Sobhy
}

\author{
Wael Mohamed Khedr
}

\begin{abstract}
Automatic diagnosis of breast cancer is an important, that's really real-world medical problem. This paper aims to describe an intelligent procedure based on fuzzy logic techniques and medical model to detect and diagnose Breast. The system has 7 input parameters and 1 output, in which the inputs are Age, Genetic Factor, Menarche Age, First Pregnancy, Menopause Age, Nutrition Habit, Life Style and the output parameter which is based on diagnosis risk degree. We have used Mamdani inference engine to deduce from the input parameters to stage the cancer.
\end{abstract}

\section{Keywords}

Fuzzy Logic, Fuzzy Inference Systems(FIS),Breast Cancer, risk analysis.

\section{INTRODUCTION}

Breast cancer is the commonest malignancy in Egyptian females. According to the data of national Cancer Institute of Cairo University it accounts for about $35 \%$ of total malignancies in females. At the same time, it is also among the most curable cancer types if it can be diagnosed early. The human brain can reasons with imprecise environments or decision based on uncertain or value judgment like the air is fine or the speed is fast or facts that are partially true. The fuzzy logic is a branch of machine intelligence that help computers to process and utilize vague data of humanistic systems. Fuzzy inference systems (FIS) are information processing systems using fuzzy logic mechanism to represent the human reasoning process and to make decisions based on uncertain, imprecise environments. FIS consists of four parts fuzzfier, rules, inference engine, and defuzzfier figure 1 .

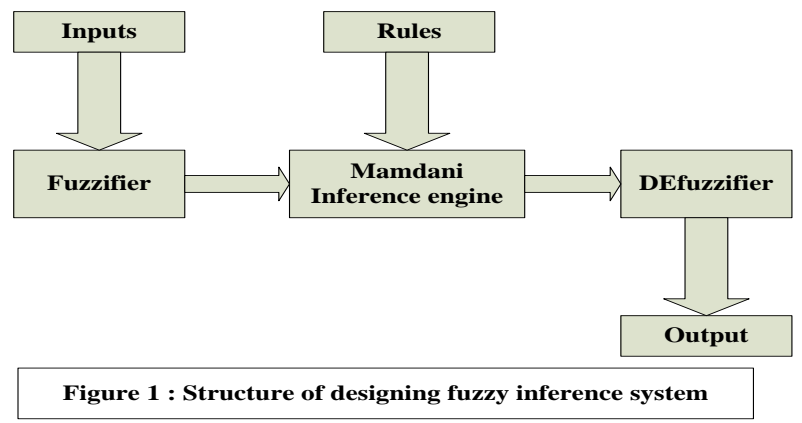

To design FIS needed the perioral experiences of human experts about field of research or knowledge base that observed and collected from operations of systems. Fuzzy logic manipulates such imprecise and vague data as fine or fast help engineering to controls and describes systems using commonsense rules that refer to indefinite quantities. So that it is possible to transition from one rule to another as the input is varied smoothly. These rules are linguistically natural representation of human's (or expert's) Knowledge base, that provides easy understanding knowledge representing scheme for explain information that has been learnt by a computer.

Fuzzy based inference mechanism is used to correlate different parameters to a common symptom. This result creates a decision support system for disease diagnosis system. Fuzzy logic system structure consists of database or prior knowledge that have to be crisp value to allow fuzzification using membership function, fuzzy control that manage fuzzification, rules evaluation, and defuzzification the output that also is crisp values. Noting also that Clinical Oncologists make diagnostic decisions about breast cancer patients based on past professional experience and knowledge, intelligent techniques are possibly the only class of automatic techniques powerful enough to emulate the expert's choice. Due to their stable behavior in the presence of noise, imprecision and uncertainty, these techniques could potentially obtain better results than classical methods. Created to substitute human-like, natural biological, nonlinear thinking in the computerized world, intelligent techniques are the most advanced modeling techniques that can evaluate and decide based on an inference process that is similar to human thinking and judgment. The most widely applied intelligent techniques are fuzzy techniques, neural network techniques, genetic algorithms and knowledge based systems (known also as expert systems).

\section{BREAST CANCER}

The diagnostic procedure used for breast cancer identification consists of appropriate observation of the imagery results in search of characteristics that fully define, according to the expert oncologist, the cancerous development stage of the detected lesion A fuzzy logic technique for the prediction of the risk of breast cancer based on a set of judiciously chosen fuzzy rules utilizing has 7 input parameters and 1 output, in which the inputs are Age, Genetic Factor, Menarche Age, First Pregnancy, Menopause Age, Nutrition Habit, Life Style and the output parameter which is based on diagnosis risk degree figure 2 .

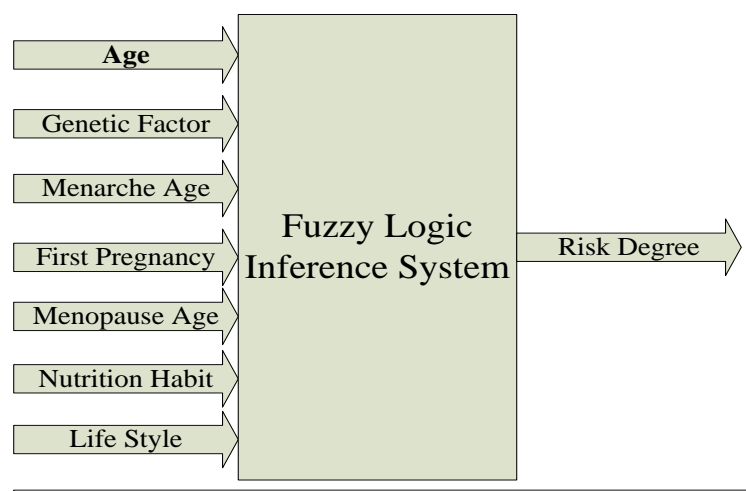

Figure 2 : Brest Cancer fuzzy logic inference system 


\section{FUZZY MEMBERSHIP FUNCTION}

The membership function editor in Fuzzy tool box is used to define the shapes of all membership functions associated with each membership variable. In that system for each of the input and output variables the membership function is defined as follows:

\subsection{Input Variables}

\subsubsection{Age}

Carcinoma of the breast is extremely rare below the age of 20 , the mean age of affection is 60 years, by the age of 90 years nearly $20 \%$ of the females are affected and the incidence of its occurrence increases through life. Age represented by three member functions young, middle and old as in table (1).

Table (1) : classification of Age

\begin{tabular}{|c|c|c|}
\hline Input field & Range & Fuzzy set \\
\hline \multirow{3}{*}{ Age } & $0-30$ & young \\
\cline { 2 - 3 } & $20-60$ & middle \\
\cline { 2 - 3 } & $50-100$ & old \\
\hline
\end{tabular}

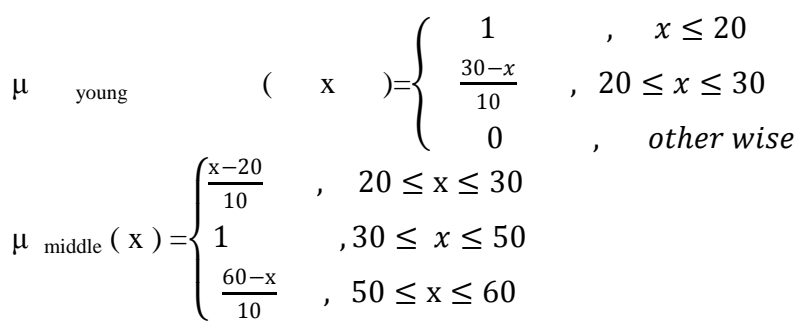

$\mu_{\text {old }}(\mathrm{x})=\left\{\begin{array}{lc}\frac{\mathrm{x}-50}{10} & , \quad 50 \leq x \leq 60 \\ 1, & x \leq 100 \\ 0 & , \text { other wise }\end{array}\right.$

\subsubsection{Genetic Factor:}

It has been proven that $5-10 \%$ of breast cancers are due to mutation in suppressor genes, in the mother or sister the risk increase by 2.3 times and in both the mother and sister the risk increase 14 times. Genetic Factor are represented by three membership functions non, far and $1^{\text {st }}$ degree as in table (2).

Table (2) : Classification of Genetic Factor

\begin{tabular}{|c|c|c|}
\hline Input field & Range & Fuzzy set \\
\hline \multirow{3}{*}{ Genetic Factor } & $0-0.7$ & None \\
\cline { 2 - 3 } & $0.5-1.5$ & Far \\
\cline { 2 - 3 } & $1.1-2$ & $1^{\text {st }}$ degree \\
\hline
\end{tabular}

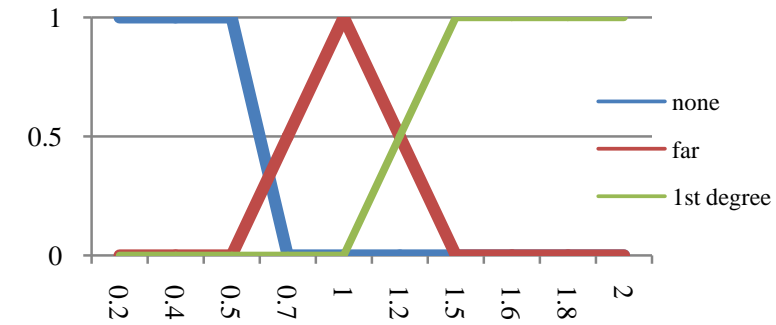

$\mu \quad$ none $\quad(x)=\left\{\begin{array}{cl}1, & x \leq 0.5 \\ 0.6(x-0.7), & 0.5 \leq x \leq 0.7 \\ 0, & \text { other wise }\end{array}\right.$ $\mu_{\text {far }} \quad(\quad \mathrm{x} \quad)= \begin{cases}2(x-0.5) & , 0.5 \leq x \leq 1 \\ 2(1.5-x) & , 1 \leq x \leq 1.5\end{cases}$

$\mu \quad$ 1st degree $(x)=\left\{\begin{array}{cc}2(x-1) & , 1 \leq x \leq 1.5 \\ 1 & , 1.5 \leq x \leq 2\end{array}\right.$

\subsubsection{Menarche Age:}

The period between the menarche and the onset of pregnancy plays a role in developing cancer breast, the longer this period, the more the chance of developing breast cancer, so risk increases it. Menarche Age is represented by two membership functions as in table (3).

Table (3) : Classification of Menarche Age

\begin{tabular}{|c|c|c|}
\hline Input field & Range & Fuzzy set \\
\hline \multirow{2}{*}{ Menarche Age } & $0-16$ & Early \\
\cline { 2 - 3 } & $13-20$ & Normal \\
\hline
\end{tabular}

$\mu \quad$ early $(x)=\left\{\begin{array}{cc}1 & , \quad x \leq 13 \\ \frac{16-\mathrm{x}}{3} & , 13 \leq x \leq 16 \\ 0 & , \quad \text { other wise }\end{array}\right.$
$\mu \quad$ normal $(x)=\left\{\begin{array}{cc}\frac{x-13}{3} & , 13 \leq x \leq 16 \\ 1 & , 16 \leq x \leq 20 \\ 0 & , \text { other wise }\end{array}\right.$

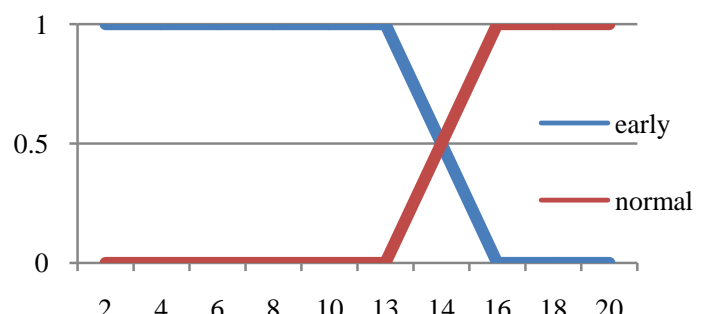

\subsubsection{First Pregnancy:}

The first pregnancy occurs after the age of 30 therefor the nulliparous women are at more risk. First Pregnancy is represented by two membership functions as in table (4).

Table (4) : Classification of Electric Volt (EV)

\begin{tabular}{|c|c|c|}
\hline Input field & Range & Fuzzy set \\
\hline \multirow{2}{*}{ First Pregnancy } & $15-30$ & Normal \\
\cline { 2 - 3 } & $28-40$ & Late \\
\hline
\end{tabular}

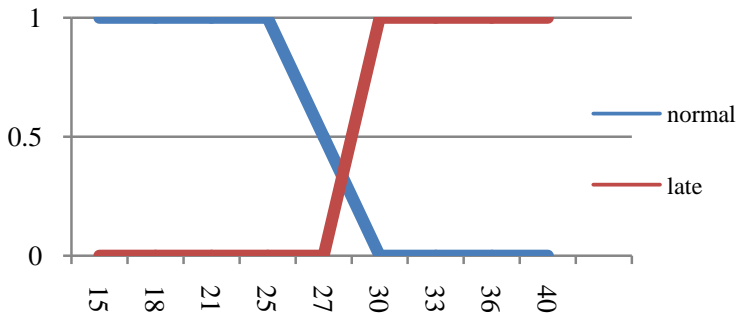


$\mu \quad$ normal $(\mathrm{x})=\left\{\begin{array}{cc}1 & , 15 \leq x \leq 25 \\ \frac{30-x}{5} & , 25 \leq x \leq 30 \\ 0 & , \quad \text { other wise }\end{array}\right.$

$\mu \quad$ late $(\mathrm{x})=\left\{\begin{array}{cc}\frac{\mathrm{x}-27}{3} & , 27 \leq x \leq 30 \\ 1 & , 30 \leq x \leq 40 \\ 0, & \text { other wise }\end{array}\right.$

\subsubsection{Menopause Age:}

Late menopause after the age of 50 increases the risk. Menopause Age is represented by two membership functions as in table (5).

Table (5) : Classification of Menopause Age

\begin{tabular}{|c|c|c|}
\hline Input field & Range & Fuzzy set \\
\hline \multirow{2}{*}{ Menopause Age } & $40-50$ & Normal \\
\cline { 2 - 3 } & $45-60$ & Late \\
\hline
\end{tabular}

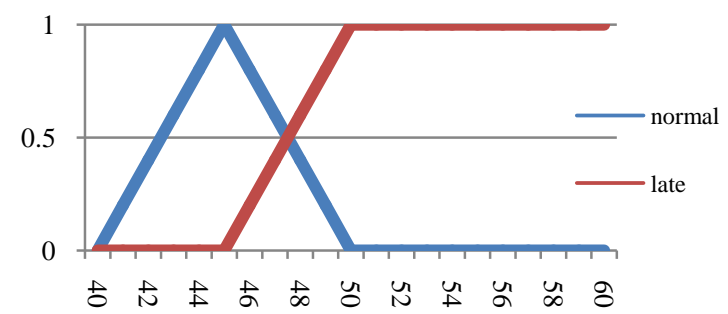

$\mu \quad$ normal $(\mathrm{x} \quad)=\left\{\begin{array}{cc}\frac{x-40}{5} & , 40 \leq x \leq 45 \\ \frac{50-x}{5} & , 45 \leq x \leq 50 \\ 0 & , \quad \text { other wise }\end{array}\right.$

$\mu \quad$ late $(\mathrm{x})=\left\{\begin{array}{cc}\frac{\mathrm{x}-50}{5} & , 45 \leq x \leq 50 \\ 1 & , 50 \leq x \leq 60 \\ 0 & \text { other wise }\end{array}\right.$

\subsubsection{Nutrition Habit:}

Women who are have bad nutrition habit as eating fast food, increase fat intake or taking imbalance meals increase risk factor of breast cancer. Nutrition habit are represented by two membership function as in table (6).

Table (6) : Classification of Nutrition Habit

\begin{tabular}{|c|c|c|}
\hline Input field & Range & Fuzzy set \\
\hline \multirow{2}{*}{ Nutrition Habit } & $0-0.7$ & Bad \\
\cline { 2 - 3 } & $0.5-1$ & Good \\
\hline
\end{tabular}

$\mu \quad$ bad $(x)=\left\{\begin{array}{cc}1 & , 0.1 \leq x \leq 0.4 \\ \frac{0.7-x}{0.3} & , 0.4 \leq x \leq 0.7 \\ 0 & , \quad \text { other wise }\end{array}\right.$

$\mu \quad \operatorname{good}(\mathrm{x})=\left\{\begin{array}{cc}\frac{\mathrm{x}-0.5}{0.2} & , 0.5 \leq x \leq 0.7 \\ 1 & , 0.7 \leq x \leq 1 \\ 0 & , \text { other wise }\end{array}\right.$

\subsubsection{Life Style:}

Smoking and alcohol intake increase risk factor and women who are physically inactive have an increased risk of breast cancer. Life Style represented by three membership functions none, sometimes and almost as in table (7).

Table (7) : Classification of Life Style

\begin{tabular}{|c|c|c|}
\hline Input field & Range & Fuzzy set \\
\hline \multirow{3}{*}{ Life Style } & $0-0.3$ & None \\
\cline { 2 - 3 } & $0.2-0.6$ & Sometimes \\
\cline { 2 - 3 } & $0.5-1$ & Almost \\
\hline
\end{tabular}

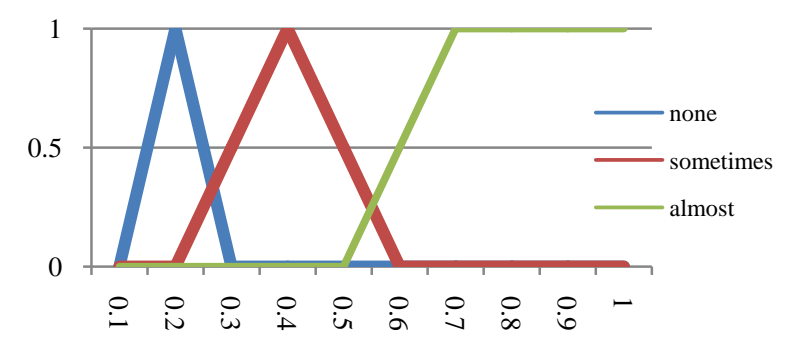

$\mu \quad$ none $\quad(\mathrm{x})=\left\{\begin{array}{cc}\frac{x-0.1}{0.1} & , 0.1 \leq x \leq 0.2 \\ \frac{0.2-x}{0.1} & , 0.2 \leq x \leq 0.3 \\ 0, & \text { other wise }\end{array}\right.$

$\mu_{\text {sometimes }}(\mathrm{x})= \begin{cases}\frac{x-0.2}{0.2} & , 0.2 \leq x \leq 0.4 \\ \frac{0.4-x}{0.2} & , 0.4 \leq x \leq 0.6\end{cases}$

$\mu_{\text {almost }}(\mathrm{x})=\left\{\begin{array}{cl}\frac{x-0.5}{0.2} & , \quad 0.5 \leq x \leq 0.7 \\ 1 & , 0.7 \leq x \leq 1\end{array}\right.$

\subsection{Output membership function}

3.2.1 Risk Degree:

Risk degree represented by four membership functions healthy, low risk, risky and extremely risk as in table (8).

Table (8) : Classification of Risk Degree

\begin{tabular}{|c|c|c|}
\hline Input field & Range & Fuzzy set \\
\hline \multirow{4}{*}{ Risk Degree } & $0-30$ & Healthy \\
\cline { 2 - 3 } & $25-55$ & Low risk \\
\cline { 2 - 3 } & $50-80$ & Risky \\
\cline { 2 - 3 } & $75-100$ & Extremely \\
\hline
\end{tabular}

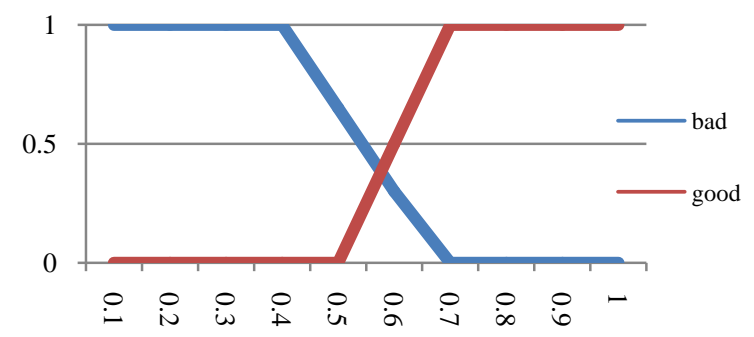




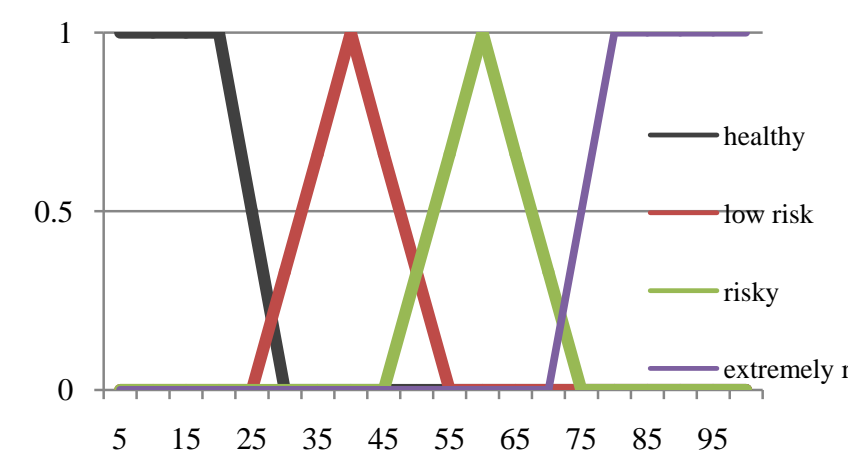

$\mu$ healthy $(\mathrm{x})=\left\{\begin{array}{cc}1 & , 0 \leq x \leq 20 \\ \frac{30-x}{10} & , 20 \leq x \leq 30 \\ 0 & , \text { other wise }\end{array}\right.$

$\mu \quad$ low risk $(\mathrm{x})=\left\{\begin{array}{cc}\frac{x-25}{15} & , 25 \leq x \leq 40 \\ \frac{55-x}{15} & , 40 \leq x \leq 55 \\ 0 & , \text { other wise }\end{array}\right.$

$\mu \quad$ risky $\quad(\mathrm{x})=\left\{\begin{array}{cc}\frac{x-45}{15} & , 45 \leq x \leq 60 \\ \frac{75-x}{15} & , 60 \leq x \leq 75 \\ 0 & , \text { other wise }\end{array}\right.$

$\mu \quad$ extremely risk $(x)=\left\{\begin{array}{cc}\frac{x-70}{10} & , 70 \leq x \leq 80 \\ 1 & , 80 \leq x \leq 100 \\ 0 & , \text { other wise }\end{array}\right.$

\section{FUZZY RULE BASE}

Fuzzy rules referred to as the IF-THEN deductive rule base form. And rules are based on natural language representation and models which are themselves based on fuzzy sets and fuzzy logic. It typically expressed inference like if the fact (premise, hypothesis, and antecedent), then infer, or derive another fact called a conclusion. It designs manual by a user, or automatic. The rules are defined by selecting the right sequence in the If-then sequence. It represents human empirical and heuristic knowledge in our language of communication that can be represented by fuzzy sets and logical connectivity of these sets. fuzzy rules utilizing has 7 input parameters and 1 output, in which the inputs are Age, Genetic Factor, Menarche Age, First Pregnancy, Menopause Age, Nutrition Habit, Life Style and the output parameter which is based on diagnosis risk degree Fuzzy rules system are shown in table (9)

Table (9) : Fuzzy rules base

\begin{tabular}{|c|c|c|c|c|c|c|c|c|}
\hline \multirow[b]{2}{*}{ ס } & \multicolumn{7}{|c|}{ input variables } & \multirow{2}{*}{ 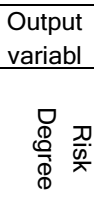 } \\
\hline & 点 & 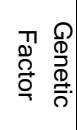 & 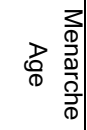 & 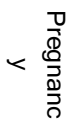 & 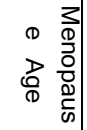 & 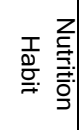 & $\begin{array}{l}\stackrel{\Gamma}{\bar{D}} \\
\frac{\mathscr{C}}{\dot{D}}\end{array}$ & \\
\hline 1 & old & $1^{\text {st }}$ & Early & None & Late & Bad & Almos & Extrem \\
\hline 2 & Old & $1^{\text {st }}$ & Early & Late & Late & Bad & Almos & Extrem \\
\hline 3 & Old & $1^{\text {st }}$ & Nor & Nor & Late & Bad & Almos & Risky \\
\hline 4 & Old & Far & Early & None & Late & Bad & Somet & Extrem \\
\hline
\end{tabular}

\begin{tabular}{|l|c|c|c|c|c|c|c|c|}
\hline 5 & Old & Far & Early & Late & Nor & Goo & Almos & Risky \\
\hline 6 & Old & Far & Nor & Nor & Nor & Goo & Somet & Low \\
\hline 7 & Old & None & Nor & None & Late & Bad & Somet & Risky \\
\hline 8 & Old & None & Nor & None & Nor & Goo & None & Low \\
\hline 9 & Old & None & Early & Late & Late & Bad & None & Risky \\
\hline 1 & Old & None & Nor & Nor & Nor & Goo & None & Healthy \\
\hline 1 & Middl & $1^{\text {st }}$ & Nor & None & Late & Bad & Somet & Extrem \\
\hline 1 & Middl & $1^{\text {st }}$ & Early & Nor & Nor & Goo & None & Low \\
\hline 1 & Middl & Far & Nor & None & Nor & Bad & Almos & Risky \\
\hline 1 & Middl & Far & Early & Late & None & Goo & None & Risky \\
\hline 1 & Middl & None & Nor & Nor & None & Goo & None & Healthy \\
\hline 1 & Youn & $1^{\text {st }}$ & Nor & None & None & Goo & None & Low \\
\hline 1 & Youn & Far & Nor & Nor & None & Bad & Somet & Low \\
\hline 1 & Youn & None & Nor & Nor & None & Goo & None & Healthy \\
\hline
\end{tabular}

\section{FUZZY LOGIC ALGORITHM}

Fuzzy logic algorithm is an algorithm that solves the problems expressed in the basic IF-THEN rule format. It consists of four steps as following:

Step 1 : linguistic Variables are the input variables of the system whose values are words or sentences from a natural language, instead of numerical values. A linguistic variable is generally decomposed into a set of linguistic terms.

Membership Functions Membership functions are used in the fuzzification and defuzzification steps of a FLS, to map the non-fuzzy input values to fuzzy linguistic terms and vice versa. A membership function is used to quantify a linguistic term.

Fuzzy Rules In a FLS, a rule base is constructed to control the output variable. A fuzzy rule is a simple IF-THEN rule with a condition and a conclusion.

Step 2 : Fuzzification means adding uncertainty by design to crisp sets or to sets that are already fuzzy and spreading the information provided by a crisp number or symbol to its vicinity so that the close neighborhood of the crisp number can be recognize by the computational tools.

Step 3:Inference For each rule which represented in fuzzy level as set of restriction on the output based on certain conditions of the input. That restriction modeled by fuzzy set and relations and connected by linguistic connections like and, or, not and else, Obtaining the output which is a new fuzzy set which is the conclusion of rule since an implication operator is applied to the value of the antecedent obtained.

Step 4: defuzzification is the process of converting the result in fuzzy set form to a crisp result. It is important process for hardware application which process based on crisp data exchange. There is not theory to justify behavior of exchange other than commonsense reasoning such that the defuzzified output must represent a weight, voted, or must suitable solution. There are two main mechanism centroid method which based on finding a balance point of a property and maxima method which based in search for the highest pack whereas

\section{BREAST CANCER SYSTEM IMPLEMENTATION \\ 6.1 Fuzzy Base Class}

This paper use Mamdani fuzzy system for the illustration which uses max aggregation and centroid method for deffuzification. The FIS Editor defines the Fuzzy Base Classes, the various inputs, i.e. Age, Genetic Factor, Menarche Age, First Pregnancy, Menopause Age, Nutrition Habit, Life Style and Risk Degree as shown in Fig 


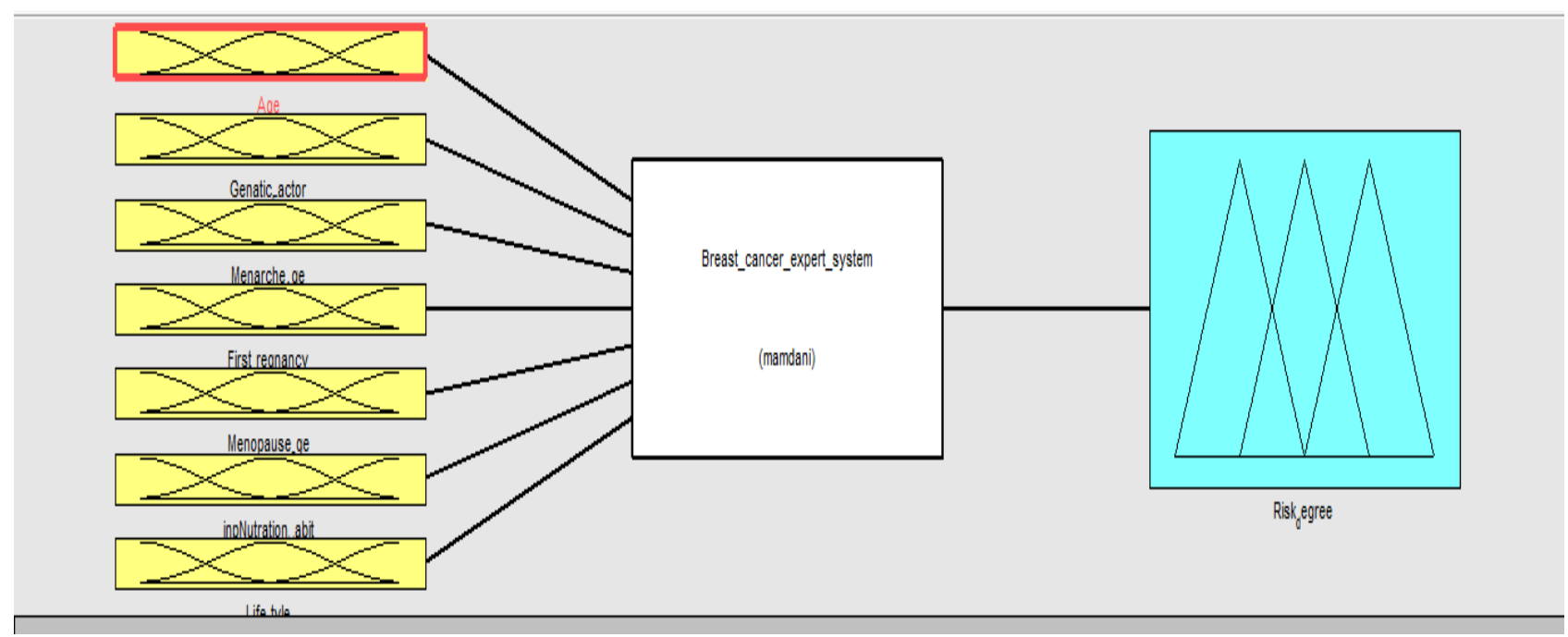

\subsection{Fuzzy Rule Base}

Fuzzy rules can be designed manually by a user, or automatically, i.e. the Rule Editor generates rules for all combinations of selected input variable and a user fills consequent fuzzy terms. Inputs and sub-sequentially provide the fuzzy outputs [10] as shown in fig .

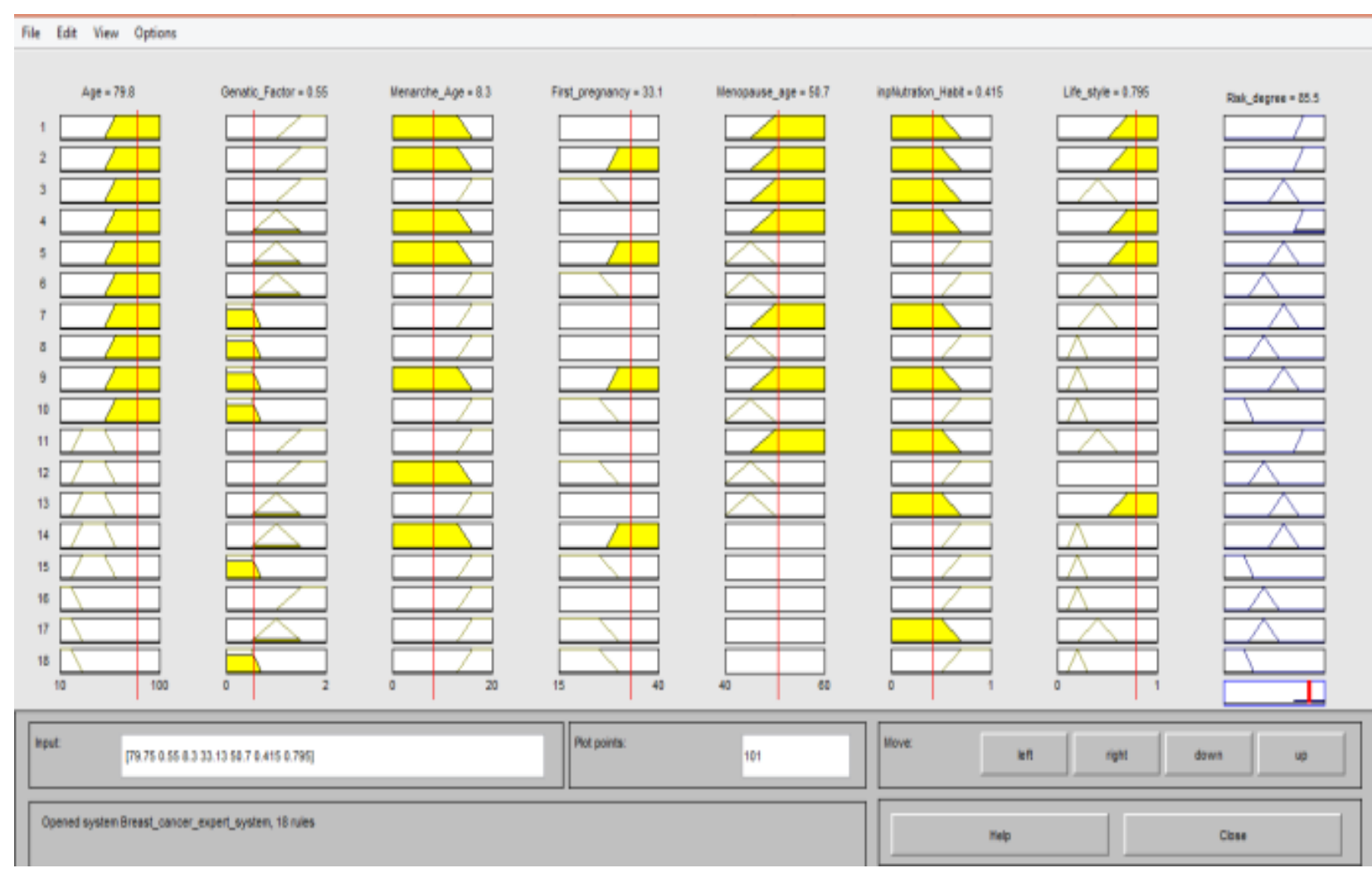

\section{CONCLUSIONS AND FUTURE WORK}

By adding intelligence to the system like fuzzy logic which is dealing with problems that are difficult and complex to study analytical that is now easy to solve in terms of linguistic variables. With most of the problems encountered in day to day life falling in this category, like washing machines, vacuum cleaners, etc., fuzzy logic is sure to make a great impact in human life. The neural net acts like computer because it maps input to output the neurons and synapses may be silicon component or equations in software that simulate their behavior. Supervised networks tune the rules of fuzzy system as if they were synapses. We will use neural network that can help fuzzy systems learn rules which can accepts pairs of input and output data and cluster them in a small number of classes. 


\section{REFERENCES}

[1] Andrew Hamilton-Wright, Daniel W.Stashuk, Transparent Decision Support Using Statistical Reasoning and Fuzzy Inference. IEEE Transactions on knowledge and Data Engineering vol.18 no.8 August 2006.

[2] Chang-Shing Lee, Mei-Hui Wang, "A Fuzzy Expert System for Diabetes Decision Support Application", IEEE Transactions on systems, man and cybernetics-part B: cybernetics, vol.41,no.1 february 2011 .

[3] Johnnie W. Huang and Rob J. Roy, "Multiple-Drug Hemodynamic Control Using Fuzzy Decision Theory," IEEE Transactions on Biomedical Engineering, vol.45, no.2, February 1998.

[4] Juan E. Moreno, Oscar Castillo, Juan R. Castro, Luis G. Martínez, Patricia Melin,"Data Mining for extraction of fuzzy IF-THEN rules using Mamdani and TakagiSugeno-Kang FIS", Engineering Letters, 15:1, EL_15_1_13 15 August 2007.

[5] K Premalatha and A M Natarajan, "A New Approach for Data Clustering Based on PSO with Local Search," Computer and Information Science, vol. 1, no. 4, 2008, pp. 139-145.

[6] Markos G. Tsipouras, Costas Voglis, and Dimitrios I. Fotiadis. "A Framework for Fuzzy Expert System Creation-Application to Cardiovascular Diseases", IEEE Transaction on Biomedical Engineering, vol.54, no.11, November 2007.

[7] Markos G. Tsipouras, Dimitrios I. Fotiadis, Themis P. Exarchos, Anna P. Kotsia, Konstantinos V. Vakalis, Katerina K. Naka, and Lampros K. Michalis, "Automated Diagnosis of Coronary Artery Disease Based on Data Mining and Fuzzy Modeling",IEEE Transactions on Information Technology in Biomedicine, vol.12,no.4,july 2008.

[8] Mei-Hui Wang and Chang-Shing Lee, Huan-Chung Li and Wei-Min Ko, "Ontology-based Fuzzy Inference Agent for Diabetes Classification", 1-4244-12145/07/\$25.00 @B2007 IEEE.

[9] R. I. John and P. R. Innocent, "Modelling Uncertainty in Clinical Diagnosis using Fuzzy logic", "Modelling Uncertainty in Clinical Diagnosis using fuzzy logic",IEEE Transactions on systems ,man and cybernetics-part B cybernetics vol.35,no.6 December 2005.

[10] Edge SB, Byrd DR, Compton CC, Fritz AG, Greene FG, Trotti A, eds. AJCC Cancer Staging Manual. 7th ed. New York: Springer; 2010.
[11] Edge SB, Byrd DR, Compton CC, Fritz AG, Greene FG, Trotti A, eds. AJCC Cancer Staging Manual. 7th ed. New York: Springer; 2010.

[12] Carey LA, Perou CM, Livasy CA, et al. Race, breast cancer subtypes, and survival in the Carolina Breast Cancer Study. JAMA. Jun 72006.

[13] C, . Elmas, Artificial Intelligence Applications, Se, ckin Publishing, Ankara, 2007.

[14] A. Bilge, O. Cam, "Women's strategies for overcoming the stress and the examination of their health beliefs as important factor for cancer preclusion", Journal of Anatolian Psychiatry, Vol. 9, pp. 16-21, 2008.

[15] M.F. Abbod, D.G. Von Keyserlingk, D.A. Linkens, M. Mahfouf,"Survey of utilization of fuzzy technology in medicine and healthcare", Fuzzy Sets and Systems, Vol. 120, pp. 331-349, 2001.

[16] A. Torres, J.J. Nieto, "Fuzzy logic in medicine and bioinformatics", Journal of Biomedicine and Biotechnology 2006,pp. 1-7, 2006.

[17] N.H. Phuong, V. Kreinovich, "Fuzzy logic and its applications in medicine", International Journal of Medical Informatics, Vol. 62, pp. 165-173, 2001.

[18] R.M. Brand, D.D. Jones, H.T. Lynch, R.E. Brand, P. Watson, R. Ashwathnayaran, H.K. Roy, "Risk of colon cancer in hereditary non-polyposis colorectal cancer patients as predicted by fuzzy modeling: influence of smoking", World Journal of Gastroenterology, Vol. 12, pp. 4485-4491, 2006.

[19] H. Seker, M. Odetayo, D. Petrovic, R.N.G. Naguib, “A fuzzy logic based method for prognostic decision making in breast and prostate cancers", IEEE Transactions on Information Technology in Biomedicine, Vol. 7, pp. $114-122,2003$

[20] F. Steimann, "Fuzzy set theory in medicine", Artificial Intelligence in Medicine, Vol. 11, pp. 1-7, 1997.

[21] S. Guadarrama, S. Munoz, C. Vaucheret, "Fuzzy Prolog: a new approach using soft constraints propagation", FuzzySets and Systems, Vol. 144, pp. 127-150, 2004

[22] Yubin Yang, Shifu Chen, Hui Lin and Yukun Ye (2004) 'A Chromatic Image Understanding System for Lung Cancer Cell Identification based on Fuzzy Knowledge', Paper presented at the IEA/AIE '2004 Proceedings of the17th international conference on Innovations in applied artificial intelligence, 2004. 